\title{
Clinical Significance of Paraspinal Muscle Parameters as a prognostic factor for survival in Gastric Cancer Patients who underwent Curative Surgical Resection
}

Wankyu Eo ${ }^{1 *}$, Jungmi Kwon ${ }^{*}$, Soomin An ${ }^{\circledR}$, Sookyung Lee ${ }^{3}$, Sehyun Kim ${ }^{\natural}$, Dongwoo Nam ${ }^{5}$, Ga Young Han', Sung Il Choi ${ }^{7}$, Ho-Yeon Chung8

1. Department of Medical Oncology \& Hematology, College of Medicine, Kyung Hee University, Seoul, Republic of Korea.

2. College of Nursing Science, Kyung Hee University, Seoul, Republic of Korea.

3. Department of Clinical Oncology, College of Korean Medicine, Kyung Hee University, Seoul, Republic of Korea.

4. Graduate School, Dankook University, Yongin, Republic of Korea

5. Department of Acupuncture \& Moxibustion Medicine, College of Korean Medicine, Kyung Hee University, Seoul, Republic of Korea.

6. Department of Music, Chang Shin University, Changwon, Republic of Korea.

7. Department of Surgery, College of Medicine, Kyung Hee University, Seoul, Republic of Korea.

8. Department of Endocrinology and Metabolism, College of Medicine, Kyung Hee University, Seoul, Republic of Korea.

*These authors contributed equally to this work as co-first authors.

$\triangle$ Corresponding author: Soomin An, College of Nursing Science, Kyung Hee University, 02447, Republic of Korea; Phone: +82-10-7269-0124, E-mail: sue339@khu.ac.kr; and Sehyun Kim, Graduate School, Dankook University, Yongin 16890, Republic of Korea; Phone: +82-31-8005-2114, E-mail: kim.ssam@gmail.com.

(c) The author(s). This is an open access article distributed under the terms of the Creative Commons Attribution License (https://creativecommons.org/licenses/by/4.0/). See http://ivyspring.com/terms for full terms and conditions.

Received: 2020.04.03; Accepted: 2020.07.20; Published: 2020.07.31

\begin{abstract}
Background: The quantitative and qualitative skeletal muscle parameters have been proposed to predict the outcome of patients with gastric cancer. However, the evidence for their association with long-term survival is still conflicting. This study aimed to investigate the effect of paraspinal muscle parameters on overall survival (OS) and disease-free survival (DFS) in patients with gastric cancer who underwent curative resection.

Methods: Patients with stages I or II gastric cancer who underwent curative resection between October 2006 and June 2016 were identified from electrical medical records. Paraspinal muscle area and attenuation were measured at the level of the third lumbar vertebra using computerized tomography images. For the analysis of OS and DFS, proportional hazards model was used, incorporating demographic, pathologic, laboratory, and radiologic variables.

Results: This study enrolled 296 patients (192 men and 104 women). In the multivariate proportional hazards model, total gastrectomy (hazard ratio [HR], 2.65; 95\% Confidence interval $[\mathrm{Cl}], 1.36-5.19 ; p=$ $0.0044)$, neutrophil-lymphocyte ratio (NLR) (HR, 1.27; $95 \% \mathrm{Cl}, 1.06-1.51 ; p=0.0081)$, serum albumin level (HR, 0.16; 95\% Cl, 0.07-0.39; $p<0.0001)$, paraspinal muscle area adjusted for body surface area $\left(\mathrm{PMA}_{\mathrm{BSA}}\right)(\mathrm{HR}, 3.06 ; 95 \% \mathrm{Cl}, 1.65-5.67 ; p=0.0004)$, and mean attenuation in paraspinal muscle (PMMA) $(\mathrm{HR}, 3.38 ; 95 \% \mathrm{Cl}, 1.75-6.53 ; p=0.0003)$ were prognostic factors for OS. Similarly, total gastrectomy $(\mathrm{HR}, 2.11 ; 95 \% \mathrm{Cl}, 1.10-4.06 ; p=0.0243)$, NLR (HR, 1.25; 95\% Cl, 1.06-1.48; $p=0.0071)$, serum albumin level (HR, 0.22; 95\% Cl, 0.10-0.51; $p=0.0035)$, PMABSA $(\mathrm{HR}, 2.42 ; 95 \% \mathrm{Cl}, 1.34-4.37 ; p=0.0035)$, and PMMA (HR, 3.19; 95\% Cl, 1.71-5.93; $p=0.0003$ ) were prognostic factors for DFS.

Conclusions: The pretreatment paraspinal muscle parameters such as PMABSA and PMMA along with total gastrectomy, NLR, and serum albumin level could predict OS and DFS in patients with stages I or II gastric cancer who underwent curative surgical resection. Because PMA $A_{B S A}$ and PMMA are newly characterized parameters in gastric cancer, the relationship with the survival of these parameters requires further validation in further studies before they are subjected to clinical applications.
\end{abstract}

Key words: Body Composition, Tomography, Spiral Computed, Stomach Neoplasms, Prognosis 


\section{Introduction}

Stomach cancer is the third leading cause of cancer-related deaths globally. Half of all cases occur in East Asia [1], and according to the Korea Central Cancer Registry (2016) data, gastric cancer is the most frequently diagnosed malignancy in Korea [2]. Although curative resection is the primary treatment for gastric cancer, the recurrence of the disease poses a problem even in early gastric cancer patients who have previously undergone curative resection. Therefore, a novel method that accurately predicts prognosis in patients with gastric cancer is needed. The tumor-related factors, including tumor size, stage, and surgical margin status $[3,4]$, and host-related factors, including neutrophil-lymphocyte ratio (NLR) [5-8], lymphocyte-monocyte ratio (LMR) [9], plateletlymphocyte ratio (PLR) [10], and absolute monocyte and lymphocyte count prognostic score [11] are considered important for determining cancer recurrence and survival.

During diagnosis of gastric cancer, more than half of the patients exhibit some degree of malnutrition. The inability to maintain one's nutritional status is an important factor in determining morbidity and survival after surgery [12]. Therefore, identifying malnutrition and adequate pre- and postoperative interventions for maintaining nutrition may reduce this risk. Various approaches have been used to assess the nutritional status of gastric cancer patients, such as anthropometric measurements, blood markers, and measurement of energy expenditure, validated nutritional risk scores, diet history, and body composition evaluation. The body composition analysis is considered to be a reliable approach for evaluation of muscle quantity and quality in gastric cancer patients. Muscle quantity can be estimated by a variety of techniques including dual-energy X-ray absorptiometry (DEXA), bioelectrical impedance assay (BIA), computed tomography $(\mathrm{CT})$, and magnetic resonance imaging. DEXA is regarded as the most representative non-invasive method for fat-free mass measurement, but the cost of equipment, need for skilled operators, lack of portability, and exposure to ionizing radiation make clinical use difficult. The BIA is a practical, non-invasive, and an easy method to perform; however, studies that evaluated BIA gave inconsistent results. Computed tomography scan is currently considered the most accurate and non-invasive method to assess muscle mass. This approach is a regular part of the standard cancer staging; therefore, it helps avoiding additional exposure to radiation doses for measuring body composition [1]. However, body composition analysis of $\mathrm{CT}$ images requires expensive professional software and specialized staff training for accurate measurement and analysis. Moreover, there were no established guidelines regarding standard muscle parameters to be used for measurement, appropriate image planes, and proper spinal levels.

The skeletal muscle area (SMA) is performed encompassing the psoas, multifidus, erector spinae, quadratus lumborum, and abdominal wall muscles (transversus abdominus, external and internal obliques, and rectus abdominus). CT-based measurement of SMA adjusted for height squared (such as the skeletal muscle index, SMI) at the level of the third lumbar spine (L3) is reportedly considered as a main determinant of muscle quantity. According to a recent review of the impact of CT-based measurement of SMI on the clinical outcomes, low SMI was a risk factor for both long-term and short-term survival outcomes in patients with gastrointestinal tumors [13]. However, measuring SMA along the torso is difficult and time consuming. Therefore, further studies for the establishment of more convenient and accurate measurement techniques to evaluate muscle quantity are required. Muscle radiation attenuation (also known as muscle radiodensity) is a radiologic index of the muscle fat content. The advent of CT also enables explorations of changes in muscle fat avoiding invasive muscle biopsy [14]. Low muscle attenuation (MA), at the level of L3, has been reported as a poor prognostic factor for survival [15-18].

Recently, a paraspinal muscle area (PMA) adjusted for height squared (such as the paraspinal muscle index, PMI) and MA in paraspinal muscle (PMMA) at the level of L3 have been reported as independent prognostic factors for survival in patients with gastrointestinal tumors [17, 19, 20]. However, there is no available study considering the clinical significance of paraspinal muscle parameters on long-term survival outcome in localized gastric cancer patients.

Therefore, the aim of present study was to evaluate the clinical significance of paraspinal muscle parameters including PMI and PMMA at the level of L3 in stages I or II gastric cancer patients with a microscopically margin-negative resection (R0 resection).

\section{Methods}

\section{Patient selection and study design}

Patients who underwent potentially curative resection for gastric cancer between October 2006 and June 2016 in a single institution were retrospectively evaluated. This study was conducted in accordance 
with the Korean regulations and the Helsinki Declaration. The Institutional Review Board of Kyung Hee University Hospital at Gangdong approved the retrospective review of the electronic medical records. Written informed consent was waived for this study because of its retrospective nature. The inclusion criteria for patients were as follows: (i) diagnosed with primary gastric cancer by expert pathologists, according to Lauren's histological classification of gastric tumors [21]; (ii) stages I or II, according to the 7th edition of American Joint Committee TumorNode-Metastasis (TNM) classification for gastric cancer [22]; (iii) underwent extended lymph node dissection (D2 lymphadenectomy) and R0 resection; and (iv) underwent gastric resection by an experienced gastrointestinal surgeon (C.S.I) who participates in more than 50 gastric cancer resections a year.

The exclusion criteria for patients were as follows: (i) concurrent second malignancies or prior malignancies within the previous five years; (ii) human immunodeficiency virus-positive, evidence of acute infection, or concomitant autoimmune disease requiring immunosuppressive therapy at the time of surgery; (iii) stages 4 or 5 chronic kidney disease; (iv) without Korean Resident Registration Number; (v) without R0 resection; (vi) received chemotherapy, radiation, or any other treatment for gastric cancer before surgery; (vii) without preoperative abdominal computed tomography scans available for review; and (viii) underwent lumbar spinal intervention or surgery [23].

A total of 329 patients were initially enrolled, and 33 patients were excluded for the following reasons: (i) eighteen patients because of the loss of preoperative CT records or poor image quality; (ii) seven Russian patients considering ethnic difference that may have affected muscle quantity or quality parameters; (iii) three patients because gastric cancer and other malignant tumors were simultaneously diagnosed; (iv) two patients without R0 resection; (v) one patient with stage 5 chronic kidney disease; (vi) one patient with acute infection; and (vii) one patient because he had a history of surgery for lumbar disease.

\section{Clinical variables}

Records of demographic and clinical variables such as age, sex, site of tumor, size of tumor, type of gastrectomy, TNM stage, Lauren classification, lymphatic/vascular/perineural invasion, adjuvant chemotherapy, anemia, serum albumin level, NLR, LMR, and PLR were collected and analyzed. Because there were no age restrictions, elderly patients were also included in this study. Analysis of blood test results was done on tests performed within seven days before surgery. If there was more than one preoperative test result, the test result closest to the date of surgery was selected. The diagnoses of anemia in men and women were based on hemoglobin levels lower than $13 \mathrm{~g} / \mathrm{dL}$ and $12 \mathrm{~g} / \mathrm{dL}$, respectively.

\section{Body composition}

Computed tomography images taken within 30 days before surgical resection were analyzed. After identification of the L3 landmark, corresponding single axial image was extracted and saved as a DICOM image file [24]. SliceOmatic software (ver. 5.0) was used to measure the patient's body composition. Hounsfield unit (HU) threshold $(-29$ to +150$)$ was used to identify and quantify PMA and PMMA. For measurement of the PMA, the erector spinae, multifidus, psoas, and quadratus lumborum were encompassed (Fig. 1). The PMI was calculated by dividing PMA by the square of the patient's height in meters. Finally, PMMA at the level of L3 was calculated; Region of interest was characterized as all pixels within muscle HU range (-29 to $+150 \mathrm{HU})$. All the measurements were performed by an experienced nurse, and the tagged image file was reconfirmed by an experienced physician before entering it in the database. All measurements were performed under the supervision of a musculoskeletal radiologist. Before statistical analysis, muscle area and attenuation were categorized with sex-specific cutoff points.

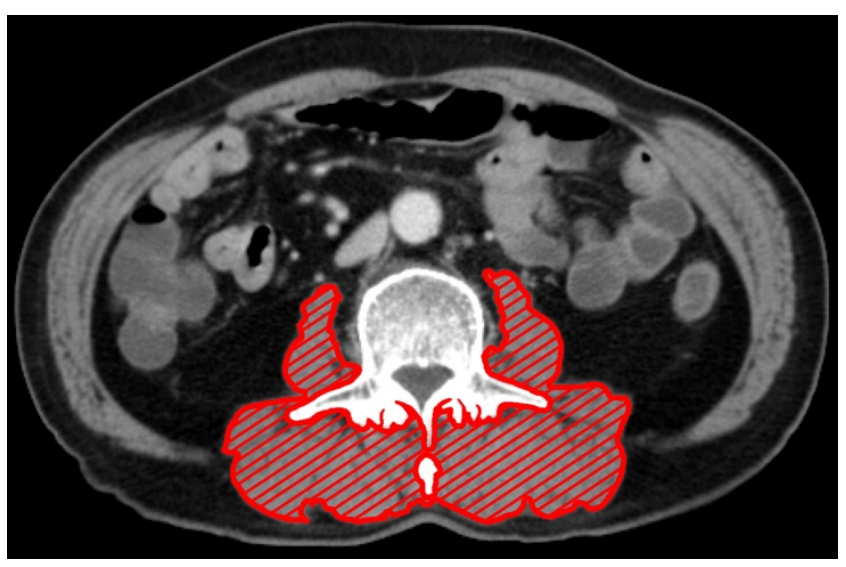

Figure 1. The cross-sectional area at L3 of paraspinal muscle (including erector spinae, multifidus, psoas, and quadratus lumborum). Paraspinal muscle is highlighted in red.

\section{Statistical analysis}

Overall survival (OS) was defined as the time from the date of surgery to the date of death or last follow-up. Disease-free survival (DFS) was defined as the time from the date of surgery to the date of relapse, death, or last follow-up. Patients who did not 
experience relapse or death were censored at the last follow-up. Curves for OS and DFS were depicted using the Kaplan-Meier method, and differences between survival curves were tested for statistical significance using the log-rank test. The continuous variables without well-known cutoff point such as size of tumor, NLR, LMR, PLR, height, and body weight were dichotomized using $\mathrm{R}$ packages (maxstat) before analysis.

The Cox proportional hazard model was used to identify the most valuable prognostic factors for OS and DFS. The continuous variables without wellknown cutoff points were not dichotomized before analysis. Only those variables that were compatible with proportional hazard assumption using the Schoenfeld residual test were analyzed using the univariate Cox model. Those variables with $p<0.05$ in the univariate Cox model were further analyzed using the multivariate Cox model. The Harrell's concordance statistics for Cox model was performed to measure discriminative capacity. The variance inflation factor (VIF) was calculated for diagnosis of multicollinearity.

All $p$-values presented were 2-sided, and statistical significance was declared at $p<0.05$. Data were analyzed using $\mathrm{R}$ packages and MedCalc (Ver. 19.2, MedCalc Software Ltd, Belgium).

\section{Results}

\section{Baseline clinical characteristics of patients}

Baseline patient characteristics are shown in Table 1 . The median age of the patients was 60 years. There were $224(75.7 \%)$ patients in stage I and 72 $(24.3 \%)$ in stage II. Total gastrectomy was performed in $51(17.2 \%)$ patients. The intestinal type by Lauren's classification was the most common type (53.4\%). Anemia was found in $89(30.1 \%)$ of the patients enrolled. The median of serum albumin level was 4.2 $\mathrm{g} / \mathrm{dL}$. The medians of NLR, LMR, and PLR were 1.9, 4.4 , and 117.2, respectively.

\section{Paraspinal muscle parameters}

There were significant correlations between PMA and height $(r=0.65, p<0.001)$, body weight $(r=$ $0.72, p<0.001)$, body surface area (BSA) $(r=0.76, p<$ $0.001)$, and body mass index (BMI) $(r=0.40, p<0.001)$ (Fig. 2). Therefore, PMA was further adjusted for body weight, BSA, and BMI forming $\mathrm{PMA}_{\mathrm{BW}}$, $\mathrm{PMA}_{\mathrm{BSA}}$, and PMA $\mathrm{BMI}$, respectively.

As there was a significant difference in the medians of PMI, PMA $\mathrm{BW}, \mathrm{PMA}_{\mathrm{BSA}}, \mathrm{PMA}_{\mathrm{BMI}}$, and PMMA between sexes $(p<0.0001$ in all variable), the threshold values of these parameters were determined with sex-specific cutoff points (Table 2).
Table 1. Characteristic of patients with gastric cancer $(n=296)$

\begin{tabular}{|c|c|}
\hline Variables & Median (IQR), or $n(\%)$ \\
\hline Age (years) & $60.0(52.0-68.0)$ \\
\hline \multicolumn{2}{|l|}{ Sex } \\
\hline Male & $192(64.1)$ \\
\hline Female & $104(35.9)$ \\
\hline \multicolumn{2}{|l|}{ Site of tumor } \\
\hline Upper & $28(9.5)$ \\
\hline Middle & $129(43.6)$ \\
\hline Lower & $136(45.9)$ \\
\hline Diffuse & $3(1.0)$ \\
\hline Size of tumor $(\mathrm{cm})$ & $2.6(1.8-4.0)$ \\
\hline \multicolumn{2}{|l|}{ TNM stage } \\
\hline I & $224(75.7)$ \\
\hline II & $72(24.3)$ \\
\hline \multicolumn{2}{|l|}{ Total gastrectomy } \\
\hline Yes & $51(17.2)$ \\
\hline No & $245(82.8)$ \\
\hline \multicolumn{2}{|l|}{ Lauren classification } \\
\hline Intestinal & $157(53.4)$ \\
\hline Diffuse & $69(23.5)$ \\
\hline Mixed & $55(18.7)$ \\
\hline Unknown & $13(4.4)$ \\
\hline \multicolumn{2}{|l|}{ Lymphatic invasion } \\
\hline No & $239(80.7)$ \\
\hline Yes & 57 (19.3) \\
\hline \multicolumn{2}{|l|}{ Vascular invasion } \\
\hline No & $290(98.0)$ \\
\hline Yes & $6(2.0)$ \\
\hline \multicolumn{2}{|l|}{ Perineural invasion } \\
\hline No & $283(95.6)$ \\
\hline Yes & $13(4.4)$ \\
\hline \multicolumn{2}{|l|}{ Adjuvant chemotherapy } \\
\hline No & $225(76.0)$ \\
\hline Yes & $71(24.0)$ \\
\hline \multicolumn{2}{|l|}{ Anemia* } \\
\hline No & 207 (69.9) \\
\hline Yes & $89(30.1)$ \\
\hline Serum albumin $(\mathrm{g} / \mathrm{dL})$ & $4.2(4.0-4.3)$ \\
\hline NLR & $1.9(1.4-2.5)$ \\
\hline LMR & $4.4(3.4-5.5)$ \\
\hline PLR & $117.2(93.6-147.4)$ \\
\hline
\end{tabular}

Abbreviations: IQR, interquartile range; TNM, tumor-node-metastasis; NLR, neutrophil-lymphocyte ratio; LMR, lymphocyte-monocyte ratio; PLR, platelet-lymphocyte ratio.

* The cutoff point is $12 \mathrm{~g} / \mathrm{dL}$ in female patients and $13 \mathrm{~g} / \mathrm{dL}$ in male patients.

Table 2. Threshold values of paraspinal muscle area adjusted for height squared, body weight, body surface area, body mass index, and mean attenuation within paraspinal muscle according to the sex

\begin{tabular}{lll}
\hline & \multicolumn{2}{l}{ Threshold values* } \\
\cline { 2 - 3 } & Male $(\mathrm{n}=192)$ & Female $(\mathrm{n}=104)$ \\
\hline PMI & 29.76 & 23.70 \\
PMA $_{\text {BW }}$ & 0.97 & 0.94 \\
PMABSA $_{\text {PMA }}$ & 38.50 & 32.41 \\
PMI $_{\text {PMMA }}$ & 2.68 & 2.05 \\
\hline
\end{tabular}

Abbreviations: PMI, paraspinal muscle index (also known as paraspinal muscle area adjusted for height squared); PMA, paraspinal muscle area; $\mathrm{PMA}_{\mathrm{BW}}, \mathrm{PMA}$ adjusted for body weight; $\mathrm{PMA}_{\mathrm{BSA}}$, PMA adjusted for body surface area; $\mathrm{PMA}_{\mathrm{BML}}$ PMA adjusted for body mass index; PMMA, mean attenuation within paraspinal muscle.

* The threshold is determined using $\mathrm{R}$ packages (maxstat).

\section{Paraspinal muscle parameters and survival}

With a median follow-up of 80.5 months (range, 0.9-145.5 months), the Kaplan-Meier method followed by the log-rank test revealed that there was a 
significant difference in OS in variables such as age $(p$ $<0.0001)$, size of tumor $(p=0.0103)$, stage $(p=0.0152)$, total gastrectomy $(p=0.0014)$, lymphatic invasion $(p=$ $0.0133)$, NLR $(p<0.0001)$, LMR $(p<0.0001), \operatorname{PLR}(p=$ $0.0277)$, anemia $(p=0.0017)$, hypoalbuminemia $(p<$ $0.0001)$, height $(p=0.0028)$, body weight $(p=0.0013)$, BSA $(p=0.0063)$, PMI $(p=0.0008), \operatorname{PMA}_{\mathrm{BW}}(p=0.0383)$, PMA $_{\text {BSA }}(p<0.0001)$, PMA $_{\text {BMI }}(p=0.0014)$, and PMMA $(p<0.0001)$ (Table 3).

Table 3. The overall survival and disease-free survival values according to the clinicopathologic variables

\begin{tabular}{|c|c|c|c|c|c|}
\hline & $n$ & 5 -year OS (\%) & $p$-value & 5-year DFS (\%) & $p$-value \\
\hline \multicolumn{6}{|c|}{ Age (years) } \\
\hline$<65$ & 181 & 94.8 & $<0.0001$ & 92.6 & $<0.0001$ \\
\hline$\geq 65$ & 115 & 79.0 & & 78.2 & \\
\hline \multicolumn{6}{|l|}{ Sex } \\
\hline Male & 192 & 86.8 & 0.3720 & 84.8 & 0.1840 \\
\hline Female & 104 & 90.9 & & 91.0 & \\
\hline \multicolumn{6}{|c|}{ Size of tumor $(\mathrm{cm})^{*}$} \\
\hline$\leq 4.5$ & 243 & 91.0 & 0.0103 & 89.3 & 0.0304 \\
\hline$>4.5$ & 53 & 78.1 & & 76.2 & \\
\hline \multicolumn{6}{|c|}{ TNM stage } \\
\hline I & 224 & 92.1 & 0.0152 & 90.7 & 0.0176 \\
\hline II & 72 & 78.3 & & 75.4 & \\
\hline \multicolumn{6}{|c|}{ Total gastrectomy } \\
\hline No & 245 & 90.7 & 0.0014 & 88.6 & 0.0060 \\
\hline Yes & 51 & 78.2 & & 78.4 & \\
\hline \multicolumn{6}{|c|}{ Lauren classification } \\
\hline Intestinal & 157 & 90.8 & 0.7730 & 86.2 & 0.9110 \\
\hline Others & 139 & 86.8 & & 87.8 & \\
\hline \multicolumn{6}{|c|}{ Lymphatic invasion } \\
\hline No & 239 & 91.6 & 0.0133 & 89.9 & 0.0383 \\
\hline Yes & 57 & 76.8 & & 75.0 & \\
\hline \multicolumn{6}{|c|}{ Vascular invasion } \\
\hline No & 290 & 89.1 & 0.1430 & 87.4 & 0.1860 \\
\hline Yes & 6 & 66.7 & & 66.7 & \\
\hline \multicolumn{6}{|c|}{ Perineural invasion } \\
\hline No & 283 & 89.6 & 0.0917 & 87.8 & 0.1340 \\
\hline Yes & 13 & 69.2 & & 69.2 & \\
\hline \multicolumn{6}{|l|}{ NLR* } \\
\hline$\leq 3.26$ & 262 & 92.4 & $<0.0001$ & 90.9 & $<0.0001$ \\
\hline$>3.26$ & 33 & 60.3 & & 57.0 & \\
\hline \multicolumn{6}{|l|}{ LMR* } \\
\hline$\leq 2.79$ & 35 & 53.0 & $<0.0001$ & 49.9 & $<0.0001$ \\
\hline$>2.79$ & 260 & 93.6 & & 92.0 & \\
\hline \multicolumn{6}{|l|}{ PLR $^{*}$} \\
\hline$\leq 188.82$ & 261 & 90.4 & 0.0277 & 88.4 & 0.0652 \\
\hline >188.82 & 34 & 78.1 & & 78.2 & \\
\hline \multicolumn{6}{|l|}{ Anemia§ } \\
\hline No & 207 & 91.8 & 0.0017 & 90.4 & 0.0032 \\
\hline Yes & 89 & 81.3 & & 79.0 & \\
\hline \multicolumn{6}{|c|}{ Hypoalbuminemia } \\
\hline No & 282 & 91.4 & $<0.0001$ & 89.6 & $<0.0001$ \\
\hline Yes & 14 & 35.7 & & 35.7 & \\
\hline \multicolumn{6}{|c|}{ Height $(\mathrm{cm})^{*}$} \\
\hline$\leq 151.0$ & 36 & 78.8 & 0.0028 & 76.8 & 0.0103 \\
\hline$>151.0$ & 260 & 90.4 & & 88.4 & \\
\hline \multicolumn{6}{|c|}{ Body weight (kg)* } \\
\hline$\leq 53.8$ & 63 & 81.8 & 0.0013 & 81.8 & 0.0068 \\
\hline$>53.8$ & 233 & 90.6 & & 88.4 & \\
\hline \multicolumn{6}{|l|}{$\operatorname{BSA}\left(\mathrm{m}^{2}\right)^{*}$} \\
\hline$\leq 1.48$ & 37 & 80.9 & 0.0063 & 80.9 & 0.0190 \\
\hline$>1.48$ & 259 & 89.9 & & 87.9 & \\
\hline \multicolumn{6}{|c|}{ BMI $\left(\mathrm{kg} / \mathrm{m}^{2}\right)$} \\
\hline$<18.5$ & 16 & 87.1 & 0.2190 & 87.1 & 0.3050 \\
\hline$\geq 18.5$ & 280 & 88.8 & & 87.0 & \\
\hline BMI $(\mathrm{kg} / \mathrm{r}$ & & & & & \\
\hline$<25$ & 190 & 86.7 & 0.1150 & 85.1 & 0.0994 \\
\hline
\end{tabular}

\begin{tabular}{llllll}
\hline & $n$ & 5 -year OS (\%) & $p$-value & 5 -year DFS (\%) & $p$-value \\
\hline$\geq 25$ & 106 & 92.2 & & 90.3 & \\
PMI $\left(\mathbf{c m}^{2} / \mathbf{m}^{2}\right) \boldsymbol{q}$ & & & & & 0.0010 \\
Low & 206 & 84.9 & 0.0008 & 83.5 & \\
High & 90 & 97.8 & & 95.2 & 0.1040 \\
PMA $_{\text {Bw }}$ & & & & & \\
Low & 65 & 82.5 & 0.0383 & 82.5 & \\
High & 231 & 90.4 & & 88.2 & \\
PMA & & & & & \\
Low & 66 & 78.4 & $<0.0001$ & 78.4 & \\
High & 230 & 91.7 & & 89.5 & \\
PMA & & & & & \\
Low & 50 & 79.8 & 0.0014 & 79.8 & \\
High & 246 & 90.6 & & 88.5 & \\
PMMA (HU) & & & & & \\
Low & 104 & 79.3 & $<0.0001$ & 76.4 & \\
High & 192 & 93.9 & & 92.8 & \\
\hline
\end{tabular}

Abbreviations: OS, overall survival; DFS, disease-free survival; TNM, tumor-node-metastasis; NLR, neutrophil-lymphocyte ratio; LMR,

lymphocyte-monocyte ratio; PLR, platelet-lymphocyte ratio; BSA, body surface area; BMI, body mass index; PMI, paraspinal muscle index (also known as paraspinal muscle area adjusted for height squared); PMA, paraspinal muscle area; PMA $_{\mathrm{BW}}$, PMA adjusted for body weight; PMA ${ }_{\mathrm{BSA}}$, PMA adjusted for BSA; PMA ${ }_{\mathrm{BMI}}$ PMA adjusted for BMI; PMMA, mean attenuation within paraspinal muscle; $\mathrm{HU}$, Hounsfield unit.

* The cutoff point is determined by using $\mathrm{R}$ packages (maxstat).

$\S$ The cutoff point is $12 \mathrm{~g} / \mathrm{dL}$ in female patients and $13 \mathrm{~g} / \mathrm{dL}$ in male patients.

I The threshold is determined with sex-specific cutoff point (Table 2).

Curves for OS and DFS were depicted using the Kaplan-Meier method, and

differences between survival curves were tested for statistical significance using the log-rank test.

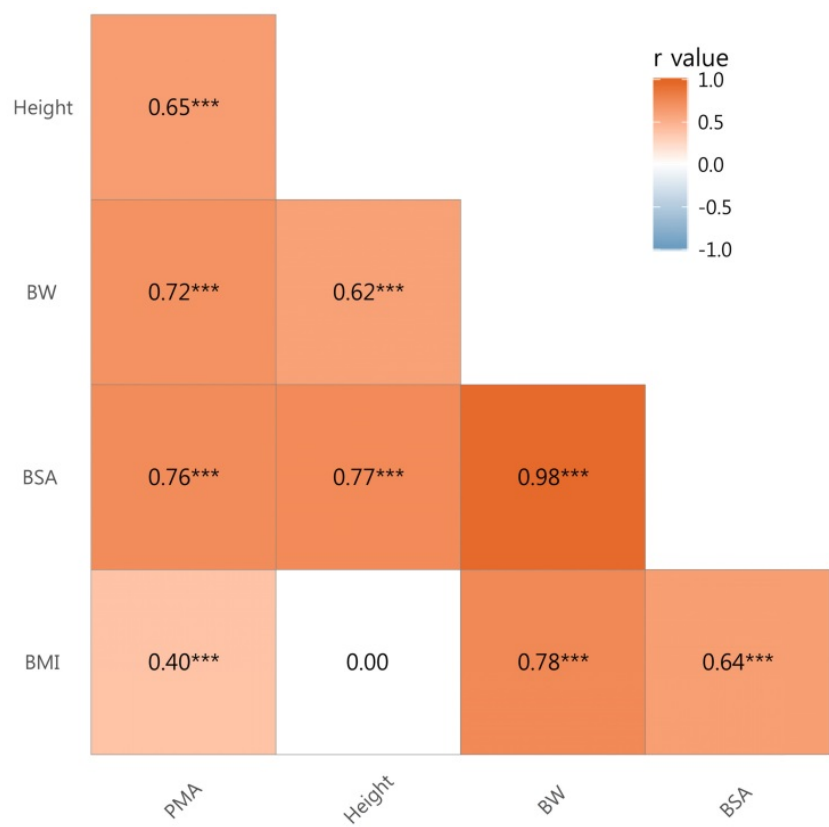

Figure 2. Correlation Coefficients by Pearson's product-moment correlation. Abbreviations: BW, body weight; BSA, body surface area; BMI, body mass index; PMA, paraspinal muscle area; ${ }^{* * *} p$ value $<0.001$.

Only those variables that were compatible with proportional hazard assumption using the Schoenfeld residual test were analyzed using the univariate Cox model. Therefore, lymphatic invasion and perineural invasion were excluded from analysis of prognostic factor for OS; in addition, lymphatic invasion, perineural invasion and BSA were excluded from analysis of prognostic factor for DFS. In the univariate Cox proportional hazards model for OS, variables 
such as age, stage, total gastrectomy, NLR, LMR, anemia, serum albumin level, PMI, PMA ${ }_{B W}, P_{B A}$, PMA $_{B M I}$, and PMMA were significant. However, using the multivariate Cox model, only variables such as total gastrectomy (hazard ratio [HR], 2.65; 95\% Confidence interval [CI], 1.36-5.19; $p=0.0044)$, NLR (HR, 1.27; 95\% CI, 1.06-1.51; $p=0.0081)$, serum albumin level (HR, 0.16; 95\% CI, 0.07-0.39; $p<0.0001$ ), PMA $_{\text {BSA }}(\mathrm{HR}, 3.06 ; 95 \% \mathrm{CI}, 1.65-5.67 ; p=0.0004)$, and PMMA (HR, 3.38; 95\% CI, 1.75-6.53; $p=0.0003$ ) were significant. The Harrell's concordance statistics for Cox model was 0.8085, indicating excellent discrimination. The VIFs for total gastrectomy, NLR, serum albumin level, PMA $A_{B S A}$, and PMMA were 1.15, $1.04,1.14,1.04$, and 1.01, respectively; therefore, there was no significant collinearity between the variables (Table 4).

Table 4. Univariate and multivariate Cox proportional hazards regression analysis of overall survival

\begin{tabular}{|c|c|c|c|c|}
\hline \multirow[t]{3}{*}{ Variable } & \multicolumn{4}{|l|}{ Overall survival } \\
\hline & \multicolumn{2}{|l|}{ Univariate } & \multicolumn{2}{|l|}{ Multivariate } \\
\hline & HR $(95 \%$ CI $)$ & $p$-value & HR $(95 \%$ CI $)$ & $p$-value \\
\hline Age (years) (<65 vs. $\geq 65)$ & $4.19(2.18-8.04)$ & $<0.0001$ & & \\
\hline Sex (Male vs. Female) & $0.74(0.39-1.43)$ & 0.3741 & & \\
\hline Size of tumor $(\mathrm{cm})$ & $1.09(0.99-1.19)$ & 0.0787 & & \\
\hline TNM stage (I vs. II) & $2.10(1.14-3.87)$ & 0.0177 & & \\
\hline Total gastrectomy (No vs. Yes) & $2.71(1.43-5.13)$ & 0.0022 & $2.65(1.36-5.19)$ & 0.0044 \\
\hline Lauren (Others vs. Intestinal) & $1.09(0.60-1.99)$ & 0.7734 & & \\
\hline Vascular invasion (No vs. Yes) & $2.77(0.67-11.45)$ & 0.1603 & & \\
\hline NLR & $1.36(1.18-1.56)$ & $<0.0001$ & $1.27(1.06-1.51)$ & 0.0081 \\
\hline LMR & $0.71(0.56-0.90)$ & 0.0048 & & \\
\hline PLR & $1.00(1.00-1.01)$ & 0.2337 & & \\
\hline Anemia (No vs. Yes)* & $2.52(1.39-4.59)$ & 0.0024 & & \\
\hline Serum albumin & $0.11(0.05-0.23)$ & $<0.0001$ & $0.16(0.07-0.39)$ & $<0.0001$ \\
\hline BSA & $0.18(0.03-1.05)$ & 0.0564 & & \\
\hline BMI & $0.92(0.83-1.01)$ & 0.0896 & & \\
\hline PMI (High vs. Low) & $5.83(1.80-18.84)$ & 0.0032 & & \\
\hline $\mathrm{PMA}_{\mathrm{BW}}$ (High vs. Low) & $1.92(1.02-3.59)$ & 0.0418 & & \\
\hline PMA $_{B S A}$ (High vs. Low) & $3.12(1.71-5.68)$ & 0.0002 & $3.06(1.65-5.67)$ & 0.0004 \\
\hline PMАвм (High vs. Low) & $2.67(1.42-4.99)$ & 0.0022 & & \\
\hline PMMA (High vs. Low) & $4.41(2.30-8.46)$ & $<0.0001$ & $3.38(1.75-6.53)$ & 0.0003 \\
\hline
\end{tabular}

Abbreviations: HR, hazard ratio; CI, confidence interval; TNM,

tumor-node-metastasis; Lauren, Lauren classification; NLR,

neutrophil-lymphocyte ratio; LMR, lymphocyte-monocyte ratio; PLR,

platelet-lymphocyte ratio; BSA, body surface area; BMI, body mass index; PMI, paraspinal muscle index (also known as paraspinal muscle area adjusted for height squared); PMA, paraspinal muscle area; $\mathrm{PMA}_{\mathrm{BW}}$, PMA adjusted for body weight; PMA $_{\mathrm{BSA}}$, PMA adjusted for BSA; PMA $\mathrm{BMI}$, PMA adjusted for BMI; PMMA, mean attenuation within paraspinal muscle.

* The cutoff point is $12 \mathrm{~g} / \mathrm{dL}$ in female patients and $13 \mathrm{~g} / \mathrm{dL}$ in male patients. The Harrell's concordance statistics for Cox model is 0.8085 , indicating excellent discrimination.

The variance inflation factors for total gastrectomy, NLR, serum albumin level, $\mathrm{PMA}_{\mathrm{BSA}}$, and PMMA are 1.15, 1.04, 1.14, 1.04, and 1.01, respectively.

Using the univariate Cox model for DFS, the same variables which proved significant in the OS analysis, except $\mathrm{PMA}_{\mathrm{BW}}$, were identified as significant. However, using the multivariate Cox model, total gastrectomy (HR, 2.11; 95\% CI, 1.10-4.06; $p=0.0243)$, NLR (HR, 1.25; 95\% CI, 1.06-1.48; $p=$ $0.0071)$, serum albumin level (HR, 0.22; 95\% CI, $0.10-$
$0.51 ; p=0.0035), \mathrm{PMA}_{\mathrm{BSA}}(\mathrm{HR}, 2.42 ; 95 \% \mathrm{CI}, 1.34-4.37$; $p=0.0035)$, and PMMA (HR, 3.19; 95\% CI, 1.71-5.93; $p$ $=0.0003$ ) were significant. The Harrell's concordance statistics for Cox model was 0.7716, indicating acceptable discrimination. The VIFs for total gastrectomy, NLR, serum albumin level, PMABSA, and PMMA were 1.14, 1.02, 1.14, 1.03, and 1.01, respectively; therefore, there was no significant collinearity between the variables (Table 5).

Table 5. Univariate and multivariate Cox proportional hazards regression analysis of disease-free survival

\begin{tabular}{|c|c|c|c|c|}
\hline \multirow[t]{3}{*}{ Variable } & \multicolumn{4}{|c|}{ Disease-free survival } \\
\hline & \multicolumn{2}{|c|}{ Univariate } & \multicolumn{2}{|l|}{ Multivariate } \\
\hline & HR $(95 \% \mathrm{CI})$ & $p$-value & $\operatorname{HR}(95 \% \mathrm{CI})$ & $p$-value \\
\hline Age (years) (<65 vs. $\geq 65$ ) & $3.45(1.89-6.31)$ & $<0.0001$ & & \\
\hline Sex (Male vs. Female) & $0.65(0.34-1.23)$ & 0.1872 & & \\
\hline Size of tumor $(\mathrm{cm})$ & $1.07(0.98-1.17)$ & 0.1468 & & \\
\hline Total gastrectomy (No vs. Yes) & $2.34(1.25-4.38)$ & 0.0076 & $2.11(1.10-4.06)$ & 0.0243 \\
\hline TNM stage (I vs. II) & $2.01(1.17-3.62)$ & 0.0200 & & \\
\hline Lauren (Others vs. Intestinal) & $0.97(0.55-1.72)$ & 0.9114 & & \\
\hline Vascular invasion (No vs. Yes) & $2.52(0.61-10.38)$ & 0.2019 & & \\
\hline NLR & $1.33(1.17-1.52)$ & $<0.0001$ & $1.25(1.06-1.48)$ & 0.0071 \\
\hline LMR & $0.69(0.55-0.87)$ & 0.0016 & & \\
\hline PLR & $1.00(1.00-1.01)$ & 0.2467 & & \\
\hline Anemia (No vs. Yes)* & $2.31(1.30-4.09)$ & 0.0042 & & \\
\hline Serum albumin & $0.14(0.06-0.28)$ & $<0.0001$ & $0.22(0.10-0.51)$ & 0.0035 \\
\hline BMI & $0.92(0.84-1.01)$ & 0.0858 & & \\
\hline PMI (High vs. Low) & $4.74(1.70-13.20)$ & 0.0029 & & \\
\hline PMA $_{\mathrm{BW}}$ (High vs. Low) & $1.65(0.90-3.06)$ & 0.1079 & & \\
\hline PMABSA (High vs. Low) & $2.61(1.46-4.65)$ & 0.0012 & $2.42(1.34-4.37)$ & 0.0035 \\
\hline PMAвмі (High vs. Low) & $2.29(1.24-4.23)$ & 0.0080 & & \\
\hline PMMA (High vs. Low) & $4.16(2.25-7.68)$ & $<0.0001$ & $3.19(1.71-5.93)$ & 0.0003 \\
\hline \multicolumn{5}{|c|}{$\begin{array}{l}\text { Abbreviations: HR, hazard ratio; CI, confidence interval; TNM, } \\
\text { tumor-node-metastasis; Lauren, Lauren classification; NLR, } \\
\text { neutrophil-lymphocyte ratio; LMR, lymphocyte-monocyte ratio; PLR, } \\
\text { platelet-lymphocyte ratio; BMI, body mass index; PMI, paraspinal muscle index } \\
\text { (also known as paraspinal muscle area adjusted for height squared); PMA, } \\
\text { paraspinal muscle area; PMA } \text { PMW }_{\text {BW }} \text { PMA adjusted for body weight; PMA }{ }_{B S A} \text { PMA } \\
\text { adjusted for BSA; PMABMI, PMA adjusted for BMI; PMMA, mean attenuation } \\
\text { within paraspinal muscle. }\end{array}$} \\
\hline \multirow{2}{*}{\multicolumn{5}{|c|}{$\begin{array}{l}\text { * The cutoff point is } 12 \mathrm{~g} / \mathrm{dL} \text { in female patients and } 13 \mathrm{~g} / \mathrm{dL} \text { in male patients. } \\
\text { The Harrell's concordance statistics for Cox model is } 0.7716 \text {, indicating acceptable } \\
\text { discrimination. }\end{array}$}} \\
\hline & & & & \\
\hline \multicolumn{5}{|c|}{$\begin{array}{l}\text { The variance inflation factors for total gastrectomy, NLR, serum albumin level, } \\
\text { PMA }_{B S A} \text { and PMMA are } 1.14,1.02,1.14,1.03 \text {, and 1.01, respectively. }\end{array}$} \\
\hline
\end{tabular}

\section{Discussions}

The aim of present study was to evaluate the clinical significance of paraspinal muscle parameters at the level of L3 in stages I or II gastric cancer patients, and we found that paraspinal muscle parameters (such as PMABSA and PMMA) could predict survival along with total gastrectomy, NLR, and serum albumin level.

In our study, paraspinal muscle was composed of multifidus, erector spinae, psoas, and quadratus lumborum as previously reported $[15,25,26]$, considering the time required for the analysis and reproducibility. However, the definition of paraspinal muscle has been somewhat heterogeneous among various studies. In studies by Dohzono and Deng, it was suggested that paraspinal muscle was composed 
of multifidus and erector spinae [19, 20]. In other studies, multifidus and erector spinae combined with either psoas muscle [27], quadratus lumborum [17], or psoas muscle/quadratus lumborum $[15,25,26]$ were defined as paraspinal muscles.

In our study, we found that PMA at the level of L3 is correlated with height, body weight, BSA, and BMI. These findings are in line with those of a previous study by Yoshizumi that showed that SMA at the level of L3 was significantly associated with the same variables as those used in our study [28]. Therefore, in our study, PMA was adjusted for height squared, body weight, BSA, and BMI forming PMI, $\mathrm{PMA}_{\mathrm{BW}}, \mathrm{PMA}_{\mathrm{BSA}}$, and $\mathrm{PMA}_{\mathrm{BMI}}$, respectively.

The role of PMI as a prognostic factor for survival has been reported recently in gastrointestinal tumors. In Hacker's study on advanced gastric and gastroesophageal junction cancers, PMI was shown to be a significant predictor for OS $(p=0.003)$ but not for DFS using the multivariate Cox model [17]. In our study, PMI was a significant prognostic factor for OS and DFS when applying univariate Cox model; however, PMI was not an independent prognostic factor for OS or DFS when the multivariate Cox model was used. It is believed that the difference in the cutoff points, the definition of paraspinal muscles, the stage of the tumor, and the location of tumor may have resulted in the inconsistent findings among studies.

Body surface area has been used to estimate metabolic rate dates since the late 19th century. Currently, cardiac output, glomerular filtration rate and pulmonary function tests, chemotherapy doses, fluid resuscitation, and calories needed are frequently expressed based on BSA [29]. In our study, there was a significant difference in OS and DFS according to BSA when applying the log-rank test. In addition, BSA was correlated with PMA, as has been reported previously [28, 30]. Therefore, we evaluated the clinical significance of PMA adjusted for BSA $\left(\right.$ PMA $\left._{\mathrm{BSA}}\right)$. Because PMA $\mathrm{BSA}_{\mathrm{B}}$ was dependent on the sex in our study, PMABSA was dichotomized with sex-specific cutoff points. In our study, PMABSA was a significant prognostic factor for both OS and DFS using the multivariate Cox model. Similarly, Chang also found that appendicular lean mass adjusted for BSA was more accurate in predicting low muscle function than are height squared- and weight-adjusted indices [30]. Because there is no available study on the clinical significance of PMABSA in gastrointestinal tumors, further studies on the value of $\mathrm{PMA}_{\mathrm{BSA}}$ is needed for validation of our findings.

Skeletal muscle contains lipid droplets within the myocytes as well as intermuscular adipocytes. The MA is a radiologic index of muscle fat content, and it is inversely related to muscle fat content [14]. When reporting MA, it is recommended to use predefined $\mathrm{HU}$ ranges to demarcate intermuscular adipose tissue (usually -190 to -30 HU) and muscle tissue (usually -29 $\mathrm{HU}$ to $+150 \mathrm{HU}$ ). In previous studies, MA has been measured in skeletal muscle areas encompassing multiple muscles along the torso (such as psoas, multifidus, erector spinae, quadratus lumborum, and abdominal wall muscles). The mean skeletal muscle attenuation (SMMA) below threshold was a poor prognostic factor for OS in patients with gastrointestinal tumors $[15,17,18]$. Therefore, SMMA is suggested to be an important biomarker for survival in gastrointestinal tumors, which still needs further studies for validation.

In our study, instead of measuring MA in skeletal muscle along the torso, we measured MA in paraspinal muscles (PMMA). In this study, there was a significant difference in median values of PMMA according to sex $(p<0.0001)$, and a significant correlation between PMMA and BMI $(r=-0.16, p=$ 0.0046). The significant correlation between MA and sex or BMI has been suggested previously $[15,17,20$, 31]. Therefore, when determining the PMMA threshold for survival, we initially planned to use both sex-and BMI-specific cutoff points as has been reported by Martin [15]. However, in a subgroup analysis, we found that there was no significant correlation between PMMA and BMI in female patients. Then, the PMMA threshold for survival was determined with sex-specific cutoff points as has been reported by Dohzono [20]. In our study, we found that PMMA was a significant prognostic factor for both OS and DFS using the multivariate Cox models. Our result is compatible with that of Dohzono who showed that lower PMMA was an independent poor prognostic factor in patients with gastrointestinal cancer with spine metastasis [20]. Therefore, measuring PMMA could be a promising alternative to measuring SMMA along the torso. However, as there are only few available studies on the clinical value of PMMA in malignancies, validation of our results by further evaluation is required.

Pathological variation in MA reflects excess fat deposition in the tissue, and is observed in people with elderly, obesity, and cancer. A poor prognosis is predicted by the presence of reduced mean MA values in patients with these conditions [14]. When dichotomizing our cohort into patients with PMMA-low and PMMA-high groups, there was a significant difference between two groups in terms of age $(p<0.0001)$, sex $(p<0.0001)$, total gastrectomy $(p=$ $0.0250)$, LMR $(p=0.0008)$, serum albumin level $(p=$ $0.0011)$, PMI $(p=0.0477)$, and PMAвм $(p=0.0222)$ (Table 6); therefore, relative older age, male 
predominance, higher total gastrectomy, lower LMR,

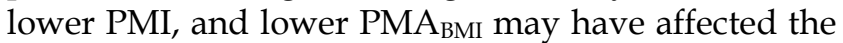
poor prognosis in PMMA-low group. Although the exact mechanism underlying the poor survival in patients with lower PMMA needs further study, in our cohort, diverse independent prognostic factors, including demographic, inflammatory, or nutritional status, may have affected the dismal prognosis in this group.

Table 6. Characteristics according to the mean attenuation within paraspinal muscles

\begin{tabular}{|c|c|c|c|}
\hline \multirow[t]{2}{*}{ Variables } & $\begin{array}{l}\text { PMMA-low group } \\
(n=104)\end{array}$ & $\begin{array}{l}\text { PMMA-high group } \\
(n=192)\end{array}$ & \multirow[t]{2}{*}{$p$-value } \\
\hline & Median (IQR), or $n(\%)$ & Median (IQR), or $n(\%)$ & \\
\hline Age (years) & $65.0(56.5-72.0)$ & $58.0(50.0-66.0)$ & $<0.0001$ \\
\hline \multicolumn{4}{|l|}{ Sex } \\
\hline Male & $93(89.4)$ & 99 (51.6) & \multirow[t]{2}{*}{$<0.0001$} \\
\hline Female & $11(10.6)$ & $93(48.4)$ & \\
\hline Size of tumor $(\mathrm{cm})$ & $2.7(2.0-4.5)$ & $2.5(1.8-3.7)$ & 0.2210 \\
\hline \multicolumn{4}{|l|}{ TNM stage } \\
\hline I & $72(69.2)$ & $152(79.2)$ & \multirow[t]{2}{*}{0.0655} \\
\hline II & $32(30.8)$ & $40(20.8)$ & \\
\hline \multicolumn{4}{|l|}{ Total gastrectomy } \\
\hline No & $79(76.0)$ & $166(86.5)$ & \multirow[t]{2}{*}{0.0250} \\
\hline Yes & $25(24.0)$ & $26(13.5)$ & \\
\hline \multicolumn{4}{|c|}{ Lauren classification } \\
\hline Intestinal & $63(60.6)$ & $94(49.0)$ & \multirow[t]{2}{*}{0.0673} \\
\hline Others & $41(39.4)$ & $98(51.0)$ & \\
\hline \multicolumn{4}{|l|}{ Lymphatic invasion } \\
\hline No & $79(76.0)$ & $160(83.3)$ & \multirow[t]{2}{*}{0.1640} \\
\hline Yes & $25(24.0)$ & $32(16.7)$ & \\
\hline \multicolumn{4}{|l|}{ Neural invasion } \\
\hline No & $97(93.3)$ & $186(96.9)$ & \multirow[t]{2}{*}{0.2330} \\
\hline Yes & $7(6.7)$ & $6(3.1)$ & \\
\hline \multicolumn{4}{|l|}{ Vascular invasion } \\
\hline No & $102(98.1)$ & 188 (97.9) & \multirow[t]{2}{*}{1.0000} \\
\hline Yes & $2(1.9)$ & $4(2.1)$ & \\
\hline NLR & $2.0(1.5-2.7)$ & $1.8(1.3-2.4)$ & 0.0525 \\
\hline LMR & $4.0(3.1-5.3)$ & $4.6(3.7-5.6)$ & 0.0008 \\
\hline PLR & $114.6(86.7-146.3)$ & $117.6(96.2-148.4)$ & 0.2530 \\
\hline \multicolumn{4}{|l|}{ Anemia§ } \\
\hline No & $68(65.4)$ & $139(72.4)$ & \multirow[t]{2}{*}{0.2329} \\
\hline Yes & $36(34.6)$ & $53(27.6)$ & \\
\hline Albumin (g/dL) & $4.1(3.9-4.3)$ & $4.2(4.0-4.4)$ & 0.0011 \\
\hline \multicolumn{4}{|l|}{ BMI $\left(\mathrm{kg} / \mathrm{m}^{2}\right)$} \\
\hline$<25$ & $61(58.7)$ & $129(67.2)$ & \multirow[t]{2}{*}{0.1630} \\
\hline$\geq 25$ & $43(41.3)$ & $63(32.8)$ & \\
\hline \multicolumn{4}{|l|}{ PMI $\left(\mathrm{cm}^{2} / \mathrm{m}^{2}\right) \Phi$} \\
\hline Low & $80(76.9)$ & $126(65.6)$ & \multirow[t]{2}{*}{0.0477} \\
\hline High & $24(23.1)$ & $66(34.4)$ & \\
\hline \multicolumn{4}{|l|}{ РМАвш } \\
\hline Low & $22(21.2)$ & $43(22.4)$ & \multirow[t]{2}{*}{0.8830} \\
\hline High & $82(78.8)$ & $149(77.6)$ & \\
\hline PMA $_{\mathrm{BSA}} \boldsymbol{\Phi}$ & & & \\
\hline Low & $30(28.8)$ & $36(18.8)$ & 0.0571 \\
\hline High & 74 (71.2) & $156(81.2)$ & \\
\hline PMA $_{\text {вMI }}$ & & & \\
\hline Low & $25(24.0)$ & $25(13.0)$ & 0.0222 \\
\hline High & $79(76.0)$ & $167(87.0)$ & \\
\hline
\end{tabular}

Abbreviations: PMMA, mean attenuation within paraspinal muscle; IQR, interquartile range; TNM, tumor-node-metastasis; NLR, neutrophil-lymphocyte ratio; LMR, lymphocyte-monocyte ratio; PLR, platelet-lymphocyte ratio; BMI, body mass index; PMI, paraspinal muscle index (also known as paraspinal muscle area adjusted for height squared); PMA, paraspinal muscle area; PMA $_{\mathrm{BW}}$, PMA adjusted for body weight; PMA $_{\mathrm{BSA}}$, PMA adjusted for body surface area; $\mathrm{PMA}_{\mathrm{BMI}}$, PMA adjusted for BMI.

$\S$ The cutoff point is $12 \mathrm{~g} / \mathrm{dL}$ in female patients and $13 \mathrm{~g} / \mathrm{dL}$ in male patients.

I The threshold is determined with sex-specific cutoff point (Table 2).
In our study, total gastrectomy was a significant factor for OS and DFS by multivariate Cox analysis. In a subgroup analysis, when dichotomizing our cohort into patients with total gastrectomy and partial gastrectomy, there was a significant difference between two groups in terms of age $(p=0.002)$, site of tumor $(p=0.001)$, and size of tumor $(p=0.001)$; therefore, relative older age, proximal location, and increased tumor size may have affected the poor prognosis in patients who underwent total gastrectomy. In contrast, there was no significant difference in serum albumin level or paraspinal muscle parameters (such as PMA, PMI, PMA ${ }_{B S A}$, and PMMA) between two groups; therefore, baseline nutritional parameters did not influence the clinical course of patients.

In our study, NLR was a significant prognostic factor for OS and DFS using the multivariate Cox model. Our results are compatible with Mellor's meta-analysis results; Mellor also showed that NLR was an important prognostic determinant for both OS and DFS after R0 resection of gastric cancer [32].

Serum albumin level has been considered as a significant nutritional marker for survival in gastric cancer $[8,33,34]$. In this study, we also found that serum albumin level was a prognostic factor for OS and DFS using the multivariate Cox model.

The strength of our study is that, to the best of our knowledge, this is the first study to evaluate the prognostic significance of PMMA in patients with early stage gastric cancer who underwent curative gastric resection. In addition, we evaluated the clinical significance of diverse paraspinal muscle parameters (such as PMA adjusted for body weight, BSA, and BMI) in patients with early stage gastric cancer, and this also could be the first trial. In this study, we found that two pretreatment paraspinal muscle parameters (such as PMMA and PMA ${ }_{B S A}$ ) could independently predict long-term outcomes (such as OS and DFS) along with total gastrectomy, NLR, and serum albumin level. Finally, another strength of our study is that, for consistency, we included only those patients who underwent gastric resection by an experienced gastrointestinal surgeon who participates in more than 50 gastric tumor resections a year.

This study has some limitations; hence, the results of the study should be interpreted carefully. First, this study was performed retrospectively; therefore, missing data including CT images was inevitable, and it may have affected the results. Second, as this was a retrospective study, we did not have an opportunity to provide special interventions to patients with lower than threshold level of PMABSA and PMMA to improve postoperative outcomes. Third, although both random errors and potential 
biases were controlled from planning through to implementation of the study, the lack of validation with an independent cohort is another limitation of our study. Regarding public database, we have a limitation issue to use CT images for review in Korean population. In addition, worldwide databases have an ethnicity issue. Based on the result of this study, we can perform prospective study with independent external validation group in the next step of this study.

In conclusion, we found that PMA $\mathrm{BSA}_{\mathrm{BS}}$ PMMA, total gastrectomy, NLR, and serum albumin level were significant determinants for both OS and DFS. The concordance statistics for the same covariates were excellent during discrimination for OS and acceptable during discrimination for DFS. Using VIFs, there was no significant collinearity between covariates in the Cox model for survival analysis. Because PMABSA and PMMA are newly characterized parameters in gastric cancer, the relationship with the survival of these parameters requires further validation in further studies before they are subjected to clinical applications.

\section{Acknowledgements}

This study was supported by a grant from National Research Foundation of Korea funded by the Korean government (NRF-2017M3A9E4057932).

\section{Competing Interests}

The authors have declared that no competing interest exists.

\section{References}

1. Kuwada K, Kuroda S, Kikuchi S, Yoshida R, Nishizaki M, Kagawa S, et al. Sarcopenia and Comorbidity in Gastric Cancer Surgery as a Useful Combined Factor to Predict Eventual Death from Other Causes. Ann Surg Oncol. 2018; 25: 1160-6.

2. Jung KW, Won YJ, Kong HJ, Lee ES. Cancer Statistics in Korea: Incidence, Mortality, Survival, and Prevalence in 2016. Cancer Res Treat. 2019; 51: 417-30.

3. Siewert JR, Bottcher K, Stein HJ, Roder JD. Relevant prognostic factors in gastric cancer: ten-year results of the German Gastric Cancer Study. Annals of surgery. 1998; 228: 449-61.

4. Dittmar Y, Schule S, Koch A, Rauchfuss F, Scheuerlein H, Settmacher U. Predictive factors for survival and recurrence rate in patients with node-negative gastric cancer-a European single-centre experience. Langenbecks Arch Surg. 2014.

5. Shimada H, Takiguchi N, Kainuma O, Soda H, Ikeda A, Cho A, et al. High preoperative neutrophil-lymphocyte ratio predicts poor survival in patients with gastric cancer. Gastric Cancer. 2010; 13: 170-6.

6. Lee DY, Hong SW, Chang YG, Lee WY, Lee B. Clinical significance of preoperative inflammatory parameters in gastric cancer patients. J Gastric Cancer. 2013; 13: 111-6.

7. Lee S, Oh SY, Kim SH, Lee JH, Kim MC, Kim KH, et al. Prognostic significance of neutrophil lymphocyte ratio and platelet lymphocyte ratio in advanced gastric cancer patients treated with FOLFOX chemotherapy. BMC Cancer. 2013; 13: 350.

8. Mohri Y, Tanaka K, Ohi M, Yokoe T, Miki C, Kusunoki M. Prognostic significance of host- and tumor-related factors in patients with gastric cancer. World J Surg. 2010; 34: 285-90.

9. Ma JY, Liu Q. Clinicopathological and prognostic significance of lymphocyte to monocyte ratio in patients with gastric cancer: A meta-analysis. Int J Surg. 2018; 50: 67-71.
10. Ohe Y, Fushida S, Yamaguchi T, Kinoshita J, Saito H, Okamoto K, et al. Peripheral Blood Platelet-Lymphocyte Ratio Is Good Predictor of Chemosensitivity and Prognosis in Gastric Cancer Patients. Cancer Manag Res. 2020; 12: 1303-11.

11. Eo WK, Jeong DW, Chang HJ, Won KY, Choi SI, Kim SH, et al. Absolute monocyte and lymphocyte count prognostic score for patients with gastric cancer. World J Gastroenterol. 2015; 21: 2668-76.

12. Karcz W, Gluszek S, Kot M, Matykiewicz J. Influence of nutritional treatment on the postoperative course in patients with gastric cancer. Adv Med Sci. 2006; 51: 278-82.

13. Su H, Ruan J, Chen T, Lin E, Shi L. CT-assessed sarcopenia is a predictive factor for both long-term and short-term outcomes in gastrointestinal oncology patients: a systematic review and meta-analysis. Cancer Imaging. 2019; 19: 82.

14. Aubrey J, Esfandiari N, Baracos VE, Buteau FA, Frenette J, Putman CT, et al. Measurement of skeletal muscle radiation attenuation and basis of its biological variation. Acta Physiol (Oxf). 2014; 210: 489-97.

15. Martin L, Birdsell L, Macdonald N, Reiman T, Clandinin MT, McCargar LJ, et al. Cancer cachexia in the age of obesity: skeletal muscle depletion is a powerful prognostic factor, independent of body mass index. J Clin Oncol. 2013; 31: 1539-47.

16. Barbalho ER, Gonzalez MC, Bielemann RM, da Rocha IMG, de Sousa IM, Bezerra RA, et al. Is skeletal muscle radiodensity able to indicate physical function impairment in older adults with gastrointestinal cancer? Exp Gerontol. 2019; 125: 110688.

17. Hacker UT, Hasenclever D, Linder N, Stocker G, Chung HC, Kang YK, et al. Prognostic role of body composition parameters in gastric/gastroesophageal junction cancer patients from the EXPAND trial. J Cachexia Sarcopenia Muscle. 2020; 11: 135-44.

18. Tamandl D, Paireder M, Asari R, Baltzer PA, Schoppmann SF, Ba-Ssalamah A. Markers of sarcopenia quantified by computed tomography predict adverse long-term outcome in patients with resected oesophageal or gastro-oesophageal junction cancer. Eur Radiol. 2016; 26: 1359-67.

19. Deng CY, Lin YC, Wu JS, Cheung YC, Fan CW, Yeh KY, et al. Progressive Sarcopenia in Patients With Colorectal Cancer Predicts Survival. AJR Am J Roentgenol. 2018; 210: 526-32.

20. Dohzono S, Sasaoka R, Takamatsu K, Hoshino M, Nakamura H. Prognostic value of paravertebral muscle density in patients with spinal metastases from gastrointestinal cancer. Support Care Cancer. 2019; 27: 1207-13.

21. Lauren P. THE TWO HISTOLOGICAL MAIN TYPES OF GASTRIC CARCINOMA: DIFFUSE AND SO-CALLED INTESTINAL-TYPE CARCINOMA. AN ATTEMPT AT A HISTO-CLINICAL CLASSIFICATION. Acta pathologica et microbiologica Scandinavica. 1965; 64: 31-49.

22. Santiago JM, Sasako M, Osorio J. [TNM-7th edition 2009 (UICC/AJCC) and Japanese Classification 2010 in Gastric Cancer. Towards simplicity and standardisation in the management of gastric cancer]. Cirugia espanola. 2011; 89: 275-81.

23. Hicks GE, Simonsick EM, Harris TB, Newman AB, Weiner DK, Nevitt $\mathrm{MA}$, et al. Cross-sectional associations between trunk muscle composition, back pain, and physical function in the health, aging and body composition study. J Gerontol A Biol Sci Med Sci. 2005; 60: 882-7.

24. Gomez-Perez SL, Haus JM, Sheean P, Patel B, Mar W, Chaudhry V, et al. Measuring Abdominal Circumference and Skeletal Muscle From a Single Cross-Sectional Computed Tomography Image: A Step-by-Step Guide for Clinicians Using National Institutes of Health ImageJ. JPEN J Parenter Enteral Nutr. 2016; 40: 308-18.

25. Wan Q, Lin C, Li X, Zeng W, Ma C. MRI assessment of paraspinal muscles in patients with acute and chronic unilateral low back pain. Br J Radiol. 2015; 88: 20140546.

26. Abe H, Takei K, Uematsu T, Tokura Y, Suzuki I, Sakamoto K, et al. Significance of sarcopenia as a prognostic factor for metastatic urothelial carcinoma patients treated with systemic chemotherapy. Int J Clin Oncol. 2018; 23: 338-46.

27. Thakar S, Sivaraju L, Aryan S, Mohan D, Sai Kiran NA, Hegde AS. Lumbar paraspinal muscle morphometry and its correlations with demographic and radiological factors in adult isthmic spondylolisthesis: a retrospective review of 120 surgically managed cases. J Neurosurg Spine. 2016; 24: 679-85.

28. Yoshizumi $T$, Shirabe $K$, Nakagawara $H$, Ikegami $T$, Harimoto $N$, Toshima $\mathrm{T}$, et al. Skeletal muscle area correlates with body surface area in healthy adults. Hepatol Res. 2014; 44: 313-8.

29. Gibson S, Numa A. The importance of metabolic rate and the folly of body surface area calculations. Anaesthesia. 2003; 58: 50-5.

30. Chang JS, Kim TH, Kim H, Choi EH, Kim N, Kong ID. Qualitative muscle mass index as a predictor of skeletal muscle function deficit in Asian older adults. Geriatr Gerontol Int. 2017; 17: 99-107. 
31. Stephens NA, Skipworth RJ, Macdonald AJ, Greig CA, Ross JA, Fearon $\mathrm{KC}$. Intramyocellular lipid droplets increase with progression of cachexia in cancer patients. J Cachexia Sarcopenia Muscle. 2011; 2: 111-7.

32. Mellor KL, Powell A, Lewis WG. Systematic Review and Meta-Analysis of the Prognostic Significance of Neutrophil-Lymphocyte Ratio (NLR) After R0 Gastrectomy for Cancer. J Gastrointest Cancer. 2018; 49: 237-44.

33. Lien YC, Hsieh CC, Wu YC, Hsu HS, Hsu WH, Wang LS, et al. Preoperative serum albumin level is a prognostic indicator for adenocarcinoma of the gastric cardia. Journal of gastrointestinal surgery: official journal of the Society for Surgery of the Alimentary Tract. 2004; 8: 1041-8.

34. Onate-Ocana LF, Aiello-Crocifoglio V, Gallardo-Rincon D, Herrera-Goepfert R, Brom-Valladares R, Carrillo JF, et al. Serum albumin as a significant prognostic factor for patients with gastric carcinoma. Ann Surg Oncol. 2007; 14: 381-9. 